\title{
TWITTER EN LAS CAMPAÑAS COMUNICATIVAS DE PELÍCULAS CINEMATOGRÁFICAS
}

\author{
Luis Deltell, José-Miguel Osteso y Florencia Claes
}
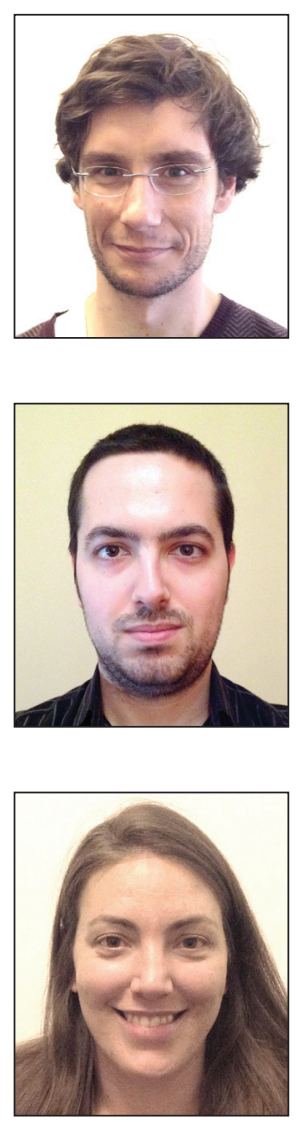

\section{Resumen}

Luis Deltell es profesor contratado doctor en la Universidad Complutense de Madrid (UCM), en el Departamento de Comunicación Audiovisual y Publicidad 1. Su campo de investigación es la historia de la imagen y el análisis de las nuevas tecnologías de las ciencias de la información. Pertenece al grupo complutense de investigación sobre cine español. Ha sido visiting scholar en Stanford University y profesor invitado en Sapienza Università di Roma y en El Cairo University. Actualmente trabaja en la medición de audiencias por medio de Twitter.

http://orcid.org/0000-0002-5230-1409

Ideltell@ccinf.ucm.es

José-Miguel Osteso es investigador en nuevas tecnologías de la información. Licenciado en comunicación audiovisual por la Univ. Complutense de Madrid. Ha trabajado como editor y jefe de posproducción en proyectos audiovisuales. Ha participado en congresos internacionales. Su campo de estudio son las redes sociales, la tecnología de la comunicación, el impacto y la creación de nuevos líderes de opinión, y la predicción electoral y la opinión política por medio de Twitter.

http://orcid.org/0000-0001-9631-7245

osteso@gmail.com

Florencia Claes trabaja en Globomedia y es investigadora y doctoranda en el Departamento de Comunicación Audiovisual y Publicidad I de la UCM. Licenciada en comunicación audiovisual con premio extraordinario en el año 2010. Su campo de estudio son las redes sociales y los líderes de opinión en espacios virtuales de España e Iberoamérica. Ha participado en congresos internacionales e investiga la relación de Twitter y el mercado audiovisual español e iberoamericano.

http://orcid.org/0000-0003-4291-0507

florencia.claes@gmail.com

Univ. Complutense de Madrid, Fac. de Ciencias de la Información Av. Complutense s/n. 28040 Madrid, España

Las campañas comunicativas de estrenos cinematográficos han experimentado una revolución cultural, social y tecnológica desde la aparición de las redes sociales. Twitter se plantea como un método para estudiar el comportamiento de la taquilla y la formación de líderes en torno a los largometrajes. Se analiza el uso de Twitter en la campaña comunicativa de una película española (Tengo ganas de ti), centrándose en los perfiles oficiales de la productora y los actores protagonistas. Además se investigan los líderes de opinión ajenos a la producción surgidos durante ésta. Para comprender el modelo de campaña comunicativa en Twitter se analizan los perfiles oficiales de cuatro películas: la ya citada, Impávido, Lobos de Arga y Els nens salvatges.

\section{Palabras clave}

Twitter, Taquilla, Líder de opinión, Red social, Internet.

\section{Title: Twitter in films communication campaigns}

\section{Abstract}

Advertising campaigns for film premieres have experienced a cultural, social and technological revolution since the dawn of social networks. Twitter is proposed as a method to study how the box office behaves and how leaders emerge among feature films. This project utilizes the Tengo ganas de ti ("I want you") case to analyze the use of Twitter in the communication campaign of a Spanish film, focusing on the producer's and lead actors' official profiles. Thought leaders unconnected with the production that emerged during the advertising campaign are also investigated. In an effort to understand Twitter as a model for communication campaigns, the official profiles of four films are analyzed: Tengo ganas de ti, Impávido, Lobos de Arga and Els nens salvatges. 


\section{Keywords}

Twitter, Box office, Thought leader, Social networks, Internet.

Deltell-Escolar, Luis; Osteso, José-Miguel; Claes, Florencia (2013). "Twitter en las campañas comunicativas de películas cinematográficas". El profesional de la información, marzo-abril, v. 22, n. 2, pp. 129-134.

http://dx.doi.org/10.3145/epi.2013.mar.05

\section{Introducción y antecedentes}

Desde el nacimiento de Twitter gran número de investigadores comprendieron que sus posibilidades para la predicción electoral, política, social y comercial serían excelentes. Así han surgido múltiples trabajos que lo relacionan con mediciones en estos campos.

Otra de las posibilidades es la predicción de los resultados de taquilla de las películas y del impacto que los líderes de opinión surgidos en ese sitio web tienen sobre la recaudación de los largometrajes. Es decir, se trata de combinar dos campos distintos de la comunicación: primero, la predicción de resultados y segundo, el impacto que manifiesta el boca a oreja electrónico, electronic word of mouth (E-WOM) y, más concretamente, el microblogging word of mouth (M-WOM) (Zhang; Skiena, 2009). Estos autores defienden cómo en la predicción de las taquillas hay que prestar atención a internet, en especial a las páginas de información cinematográfica, como IMDb (Internet movie database), y a la opinión que se genera a partir de ellas.

http://www.imdb.com

\section{El éxito de la campaña comunicativa de} Tengo ganas de ti avala que existe una relación entre el aumento de actividad en Twittery un mayor número de espectadores

En 2012 se han publicado dos modelos matemáticos de predicción de campañas comunicativas por Twitter. Se basan en estadísticas y en aspectos cualitativos distintos: el primero de ellos aborda desde la perspectiva matemática el estudio de la evolución de la taquilla, su resultado es un modelo estadístico (Ishii et al., 2012). El segundo se realiza desde el área del marketing y, por ello, se centra en una línea económica (Hennig-Thurau; Wiertz; Feldhaus, 2012).

Además de la predicción de taquilla, esta investigación pretende observar la formación de líderes en Twitter. Para entender la creación de opinión en este sitio web nos basamos en las investigaciones precedentes sobre Twitter y política (Said-Hung; Arcila-Calderón, 2011), sobre redes sociales y televisión (Ferreras-Rodríguez, 2011) y, sobre todo, el impacto de la opinión de los perfiles y usuarios de este espacio de microblogging (Marwick; Boyd, 2011).

Es significativa la repercusión de las campañas comunicativas cinematográficas entre los nativos digitales. Para entender mejor el comportamiento de este nicho de mercado utilizamos los estudios sobre adolescentes y redes sociales (Núñez-Gómez; García-Guardia; Hermida-Ayala, 2012) y nativos digitales (García-García; Gértrudix, 2009) que abordan el manejo que hacen estos usuarios.

Por último es importante consultar herramientas de medición de influencia en Twitter. Para ello, monitorizaremos el factor Klout que, a pesar de sus carencias, se perfila como el estándar de influencia en la red (Serrano-Puche, 2012).

\section{Objetivos e hipótesis}

Utilizando la literatura científica precedente, esta investigación propone analizar el caso de la campaña comunicativa de la película española Tengo ganas de ti de Fernando González Molina (2012) y la formación de líderes de opinión en Twitter sobre dicha obra. Además, se analiza el lanzamiento y promoción en este sitio web de otros tres largometrajes españoles, que sirven de ejemplos de control: Impávido de Carlos Therón (2012), Lobos de Arga de Juan Martínez Moreno (2012) y Els nens salvatges (Los niños salvajes) de Patricia Ferreira (2012). Todas ellas estrenadas en fechas próximas a Tengo ganas de ti.

Nuestros objetivos son:

- Cuantificar el impacto global de tuits e impresiones en torno a Tengo ganas de ti.

- Medir la evolución de seguidores, seguidos y del factor Klout de los actores protagonistas y perfiles promocionales de este largometraje.

- Analizar la creación de líderes de opinión sobre dicha película y su comportamiento.

- Estudiar la posible predicción de taquilla por medio de Twitter. Para ello se comparan los datos del largometraje de González Molina con las tres películas de control.

Los tres primeros objetivos se centran sólo en la película Tengo ganas de ti y no pueden ser comparados con los resultados de las obras de control, ya que éstas, como se verá, difieren en su producción y su contexto. Sin embargo, el objetivo cuarto sí es aplicable ya que la predicción de taquilla por medio de Twitter debe ser generalizable a cualquier película y promoción.

\begin{tabular}{|c|l|l|l|c|}
\hline \multicolumn{4}{|l|}{ Nombre del perfil } \\
\hline $\begin{array}{c}\text { Días de } \\
\text { control }\end{array}$ & N. seguidores & N. seguidos & N. tuits & $\begin{array}{c}\text { Factor } \\
\text { Klout }\end{array}$ \\
\hline 22 de mayo & & & & \\
\hline 23 de mayo & & & & \\
\hline$\ldots$ & & & & \\
\hline 19 de julio & & & & \\
\hline 20 de julio & & & & \\
\hline
\end{tabular}

Tabla1. Matriz de datos 


\begin{tabular}{|l|l|}
\hline \multicolumn{1}{|c|}{ Aplicación } & \multicolumn{1}{c|}{ Descripción } \\
\hline $\begin{array}{l}\text { Tweettronics } \\
\text { http://www.tweettronics.com }\end{array}$ & $\begin{array}{l}\text { Monitoriza el uso de un hashtag a lo largo de un período, durante una unidad mínima de } \\
\text { tiempo de 24 horas. }\end{array}$ \\
\hline $\begin{array}{l}\text { Hootsuite } \\
\text { http://hootsuite.com }\end{array}$ & $\begin{array}{l}\text { Gestiona redes sociales, ayuda a organizar la visualización y extracción de los datos requeridos } \\
\text { de los perfiles o hashtags seleccionados y permite extraer los tuits generados por los usuarios, } \\
\text { contabilizar los retuits sobre cada uno y ofrece el factor Klout. }\end{array}$ \\
\hline $\begin{array}{l}\text { Follow the hashtag } \\
\text { http://www.followthehashtag.com }\end{array}$ & $\begin{array}{l}\text { Muestra los 10 usuarios que más han utilizado un determinado hashtag. Unidad mínima tem- } \\
\text { poral de 24 horas. }\end{array}$ \\
\hline $\begin{array}{l}\text { HashTracking } \\
\text { https://www.hashtracking.com }\end{array}$ & $\begin{array}{l}\text { Monitoriza el uso de un determinado hashtag durante 24 horas. Muestra los usuarios que } \\
\text { más veces lo han utilizado, de ellos, los que más seguidores tienen y, por último, el número de } \\
\text { "impresiones", mostrando así su potencialidad. }\end{array}$ \\
\hline $\begin{array}{l}\text { Buscador avanzado de Twitter } \\
\text { https://twitter.com/\#!/search-advanced }\end{array}$ & $\begin{array}{l}\text { Permite una búsqueda paramétrica mediante el uso de palabras, frases, desde qué cuenta o } \\
\text { para qué cuenta, en un período de tiempo, excluir determinados items, etc. }\end{array}$ \\
\hline
\end{tabular}

Tabla 2. Aplicaciones en red

La hipótesis de partida es que el impacto de las campañas comunicativas en Twitter es relevante y justifica que las productoras y distribuidoras apuesten por este canal para publicitar sus productos. Especial interés tiene la formación de líderes de opinión de manera espontánea que interactúan sobre estas películas.

\section{Metodología}

Tengo ganas de ti adapta el libro homónimo del autor italiano Federico Moccia. Se trata de una secuela de Tres metros sobre el cielo (2010) del mismo director que adapta otro texto de dicho escritor. El guionista en ambas ocasiones fue Ramón Salazar.

Las dos películas están enfocadas a un público adolescente y principalmente femenino. Su objetivo es "llegar al público y emocionarlo", así lo describe el propio director (Fernández, 2012). Su impacto se debe a la presencia de actores icono de ese target potencial, en especial Mario Casas. Ni el director ni el guionista del largometraje han ocultado su interés por lograr un producto atractivo y popular. Efectivamente, Tres metros sobre el cielo y Tengo ganas de ti fueron grandes éxitos comerciales. La campaña de comunicación de la última entrega estuvo respaldada por Globomedia, productora de la película, Antena 3 y Charanga Films.
El estreno de la obra tuvo lugar el 22 de junio de 2012. Fue el largometraje español más visto durante su primera semana de exhibición, hasta que se presentó Lo imposible de Juan Antonio Bayona (2012).

\section{La opinión y el debate son generados y fomentados de manera eminente desde el entorno de la propia producción}

Para esta investigación se han seguido diariamente los perfiles oficiales en Twitter de la película y de los actores protagonistas: Mario Casas, Clara Lago, María Valverde, TGDT y 3 MSC. La muestra se realizó desde el día 2 de junio hasta el 2 de julio de 2012, es decir, veinte días previos y 10 días posteriores al estreno.

Para el seguimiento de cada perfil se elaboró una ficha (tabla 1), que analiza el número de seguidores que tiene el usuario, el número de perfiles que sigue, el número de tuits que emite y el factor Klout.

Uno de los aspectos fundamentales es el comportamiento del número de seguidores, que aumentó considerablemente en los cinco perfiles estudiados, lo que demuestra su im-

\begin{tabular}{|l|c|c|c|r|r|}
\hline \multicolumn{1}{|c|}{ Días de control } & @mario_casas_ & @ClaraLago1 & @mariavalverder & @TGDT & @3MSC \\
\hline 2 de junio & 777.065 & 12.317 & 149.619 & 44.793 & 39.001 \\
\hline 3 de junio & 778.895 & 12.365 & 149.841 & 44.984 & 39.210 \\
\hline$\ldots$ & $\ldots$ & $\ldots$ & $\ldots$ & $\ldots$ & $\ldots$ \\
\hline 1 de julio & 878.267 & 29.456 & 183.390 & 60.346 & 49.005 \\
\hline 2 de julio & 881.398 & 29.998 & 183.404 & 60.611 & 49.218 \\
\hline$\Delta 31$ días & 104.333 & 17.681 & 33.785 & 15.818 & 10.217 \\
\hline \% crecimiento total & 13,43 & 143,55 & 22,58 & 35,31 & 26,20 \\
\hline $\begin{array}{l}\text { Promedio nuevos } \\
\text { seguidores por día }\end{array}$ & 3.478 & 589 & 1126 & 527 & 341 \\
\hline N. de tuits generados & 50 & 71 & 36 & 68 & 217 \\
\hline $\begin{array}{l}\text { Nuevos seguidores } \\
\text { por tuit generado }\end{array}$ & 2.087 & 249 & 938 & 233 & 47 \\
\hline
\end{tabular}

pacto en Twitter. Para analizar los usuarios se midieron cuatro diferenciales: porcentaje del crecimiento total, promedio de nuevos seguidores por día, número de tuits generados y nuevos seguidores por tuit publicado.

Además se analizó el comportamiento del hashtag \#TengoGanasDeTi. Este es el propuesto de forma oficial por el equipo de comunicación de la película. Con el contador Hashtracking se monitorizó diariamente la evolución de los 10 perfiles más activos que mencionan ese hashtag. Tres datos son determinantes en este estudio: 


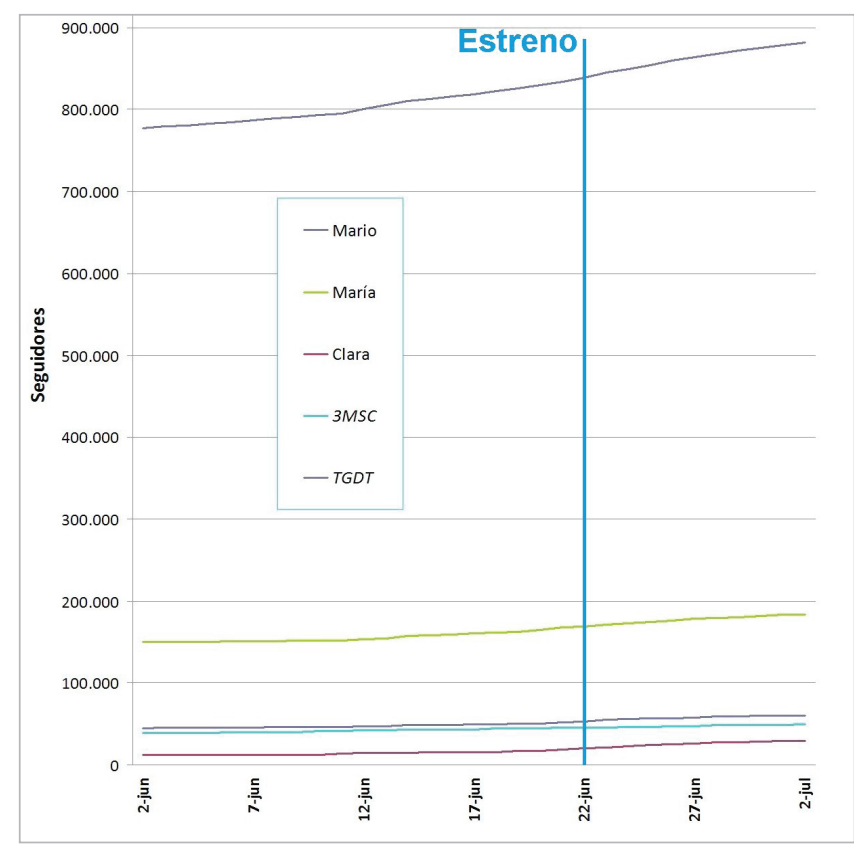

Gráfico 1. Crecimiento de seguidores

- número total de tuits escritos con dicho tema;

- impresiones;

- conocer los 10 perfiles diarios más activos.

La tabla 2 muestra las aplicaciones en red consultadas. Con estos instrumentos se monitorizaron los hashtags y perfiles implicados en el lanzamiento y promoción de los largometrajes. Estos datos se recopilaron cada 24 horas, pero cada 12 se hizo un seguimiento de control, en caso de presentar singularidades se reflejaría en las tablas.

\section{Discusión y comparación}

El modelo estadístico (Ishii et al., 2012) y el modelo de previsión económica (Hennig-Thurau; Wiertz; Feldhaus, 2012) centran su atención principal en el número de tuits generados sobre una película y atienden de forma significativa al incremento de seguidores de los perfiles oficiales de las películas y de sus protagonistas.

Para este análisis se siguió la evolución de los dos usuarios oficiales de la película y de los tres intérpretes protagonistas. De acuerdo con estos datos, tanto los perfiles encargados para la promoción directa del estreno como los de los actores aumentan significativamente en el período de estudio.

Esta primera magnitud permite comprender que la expectativa de la película por parte de los internautas es alta y, en buena medida, viene dada por el éxito del largometraje anterior. EI principal motor de la promoción es Mario Casas, que alcan- za casi los 900.000 seguidores durante el tiempo de control.

En la tabla 3 se ofrece el aumento de número de seguidores por perfil. Se indica el crecimiento total, el promedio de nuevos seguidores por día, el número de tuits generados, y el número de nuevos seguidores por cada tuit generado.

Durante la promoción de la película, los perfiles @ClaraLago1, @TGDTy @3MSC son muy activos y la cantidad de tuits emitidos por ellos aumenta. Tras el estreno de la obra, la actividad de los tres perfiles desciende considerablemente. @mariavalverdery@mario_casas_mantienen su ritmo habitual de tuits durante el tiempo de control.

Además del número de seguidores, es importante registrar la evolución del factor Klout y el número de seguidos por perfil (tabla 5).

\section{La promoción de Tengo ganas de ti supo crear una red entre todos los perfiles im- plicados en el lanzamiento, aprovechan- do la retroalimentación entre seguidores}

El incremento del número de seguidos se sitúa entre el 9 y el 20\% en todos los perfiles, salvo en el de @mario_casas_ que no varía, pero este perfil es el que presenta el mayor aumento en factor Klout y en número de seguidores. Por lo tanto, no se encontró una relación entre el aumento del factor Klout y del número de seguidores con respecto al del número de seguidos.

Los usuarios de los protagonistas ganan más seguidores diarios y aumentan más su factor Klout que los perfiles oficiales de las películas. El que más aumenta es @mario_casas_, quien se hace con 104.333 nuevos, pero su porcentaje es el menor. @ClaraLago1 es la que más gana en porcentaje (143\%), porque sus seguidores originales eran los más bajos del grupo analizado. En este sentido su factor Klout también gana, y pasa de 53 a 63 puntos, subiendo 5 puntos la semana del estreno.

El factor Klout de los 5 perfiles analizados crece durante el período estudiado y, a excepción del perfil de la película anterior (@3MSC), todos experimentan una subida más pro-

\begin{tabular}{|l|c|c|c|c|c|}
\hline \multicolumn{1}{|c|}{ Días de control } & @mario_casas_ & @ClaraLago1 & @mariavalverder & @TGDT & @3MSC \\
\hline Factor Klout 2 de junio & 73 & 53 & 62 & 58 & 59 \\
\hline 3 de junio & 73 & 54 & 62 & 58 & 59 \\
\hline & $\ldots$ & $\ldots$ & $\ldots$ & $\ldots$ & $\ldots$ \\
\hline 1 de julio & 78 & 63 & 67 & 65 & 61 \\
\hline 2 de julio & 78 & 63 & 67 & 64 & 61 \\
\hline$\Delta$ Klout 31 días & 5 & 10 & 5 & 6 & 2 \\
\hline$\Delta$ Klout/día & $+0,16$ & $+0,32$ & $+0,16$ & $+0,19$ & $+0,06$ \\
\hline Seguidos primer día & 151 & 10 & 106 & 56 & 16 \\
\hline Seguidos último día & 151 & 11 & 118 & 67 & 19 \\
\hline$\Delta$ seguidos 31 días & 0 & 1 & 12 & 11 & 3 \\
\hline
\end{tabular}

Tabla 4. Factor Klout y seguidos por perfil 
nunciada en la semana del estreno (gráfico 2).

El gráfico 2 nos muestra cómo la estrategia comunicativa de Tengo ganas de ti favorece a los perfiles analizados en el momento del estreno de la obra. Sin duda, uno de los grandes aciertos es que todos los usuarios implicados en el proyecto y en el lanzamiento se benefician mutuamente. El aumento del factor Klout de los cinco perfiles es una señal inequívoca de las expectativas sobre el film en Twitter.

Un último factor destacable es la relación entre los tuits emitidos y el número de seguidores adicionales que logran los perfiles estudiados. Otra vez, observamos que el protagonista masculino es el que alcanza los mejores resultados. @mario_casas_obtiene 2.087 seguidores por cada tuit que publica.

@mariavalverder es la que menos ha tuiteado de los 5, pero cada tuit suyo le granjea 938 seguidores nuevos. Todos los perfiles experimentan una subida a partir de los días 12 y 13 de junio, por la visita de los actores a un programa de televisión en prime time, y los días previo y posterior al estreno.

Estos datos permiten observar el inmenso flujo de movimiento que se produjo en Twitter acerca de la película Tengo ganas de ti. Muy especialmente muestran a Mario Casas como un gran polo de atracción en número de seguidores.

La promoción cinematográfica en Twitter se muestra como una estrategia útil como complemento de una campaña tradicional

\section{Monitorización del hashtag \#tengoganasdeti}

Para entender la formación de líderes de opinión sobre la película Tengo ganas de ti, se monitorizaron los 10 perfiles diarios que más veces escribían sobre el hashtag oficial, \#tengoganasdeti. Es decir, se analizaron aquellos usuarios que escribieron en Twitter sobre la obra y cuál fue el flujo de sus tuits.

Lo primero que se observa es que el número de tuits escritos por los 10 usuarios más activos con dicho hashtag aumenta hacia el día del estreno. Es precisamente en esta jornada cuando más tuits se generan (gráfico 4).

El liderazgo de opinión se basa en una relación entre la actividad de un perfil emisor y la cantidad de seguidores del mismo. Para poder medirla se utilizó el parámetro de impresiones, que surge de multiplicar el número de tuits emitidos por los seguidores del perfil emisor. Este producto permite estimar el alcance potencial de cada tuit y la capacidad de impacto de cada cuenta y mensaje. El concepto de impresión ayuda a discriminar los perfiles más seguidos de aquellos que, por baja actividad, no generan opinión.

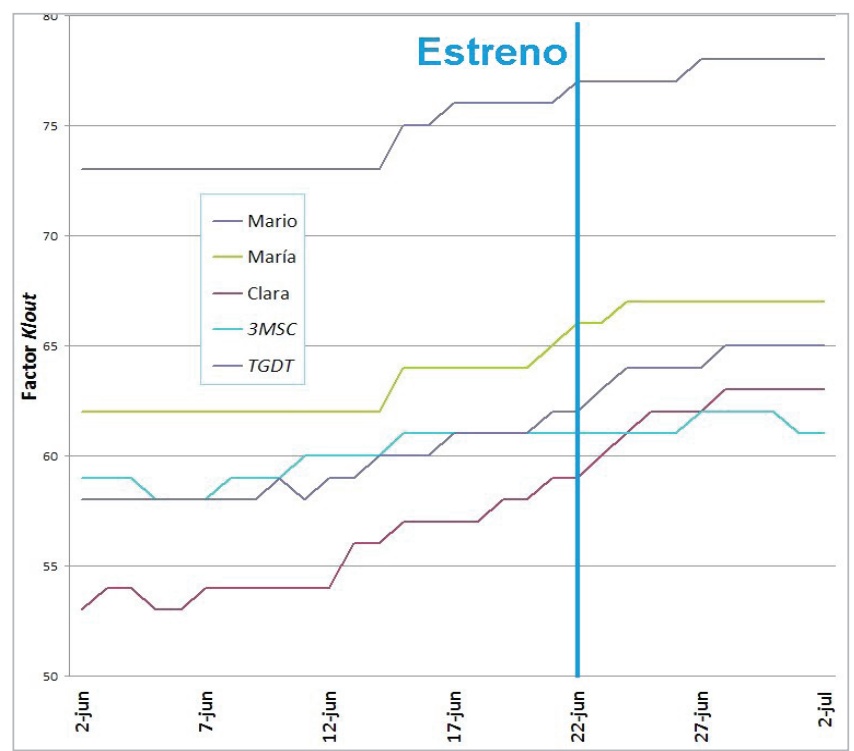

Gráfico 2. Aumento del factor Klout

La monitorización del hashtag oficial \#tengoganasdeti permite configurar una lista (tabla 5) de los 10 perfiles con mayor número de impresiones.

En el caso de \#tengoganasdeti, los perfiles con mayor número de impresiones son institucionales (I) y, salvo el primero de ellos (que corresponde a la exhibidora mexicana Cinemex), guardan una estrecha relación con la producción del filme (@otramovida es un programa de Antena 3 orientado a un target similar al de la película, @antena3com es productora de la cinta, @3MSC hace referencia a la película que precede a Tengo ganas de ti y, @lasextatv, absorbida por el grupo Antena 3, tenía parte de su accionariado en manos de Globomedia, también productora del título). De este modo se observa cómo la opinión y el debate son generados y fomentados desde el ámbito de la propia producción. De la misma forma, de los 5 perfiles personales con mayor capacidad de impacto, 3 guardan relación con la película. Esto indica que sólo 2 líderes de opinión son ajenos a la obra.

La monitorización del hashtag oficial \#tengoganasdeti permitió entender que el impacto de la película era grande. Existía un gran número de usuarios comentando y hablan-

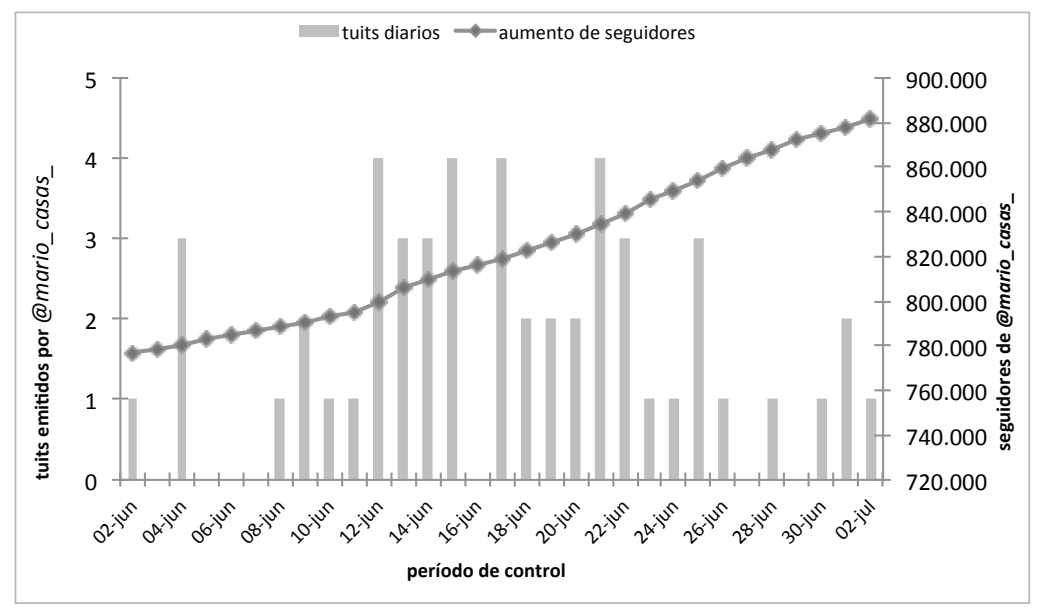

Gráfico 3. Tuits y seguidores de Mario Casas 


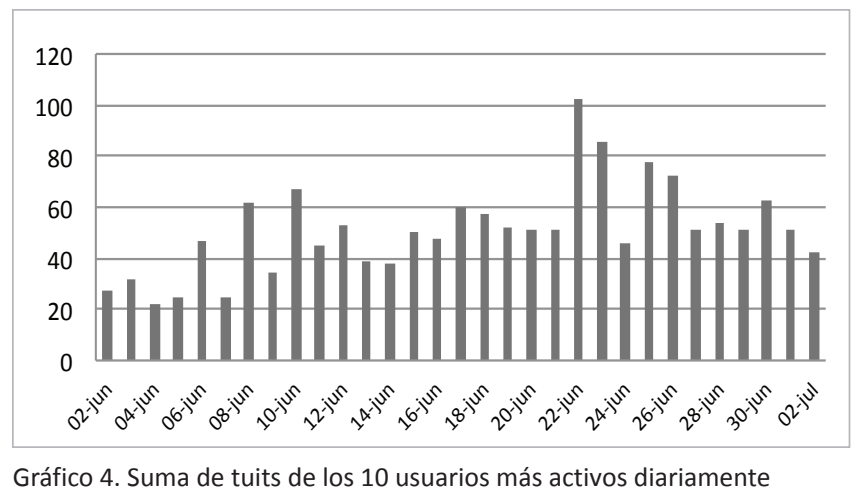

do sobre la obra. Este debate no era espontáneo, sino que estaba estimulado por la estrategia comunicativa de la productora y de los actores de la película.

\section{Datos sobre predicción de taquilla}

Para predecir el éxito comercial del largometraje con Twitter, se siguió a tres películas españolas estrenadas en ese período: Els nens salvatges, Lobos de Arga e Impávido. Todas contaban con perfil oficial en esta red. La obra de Patricia Ferreira diseñó dos usuarios oficiales, uno en lengua catalana y otro en castellano; en este caso, se estudiaron ambos perfiles y se muestra el sumatorio de los seguidores.

Es importante indicar que ninguno de estos tres largometrajes es una secuela (que sí es el caso de Tengo ganas de ti). Tampoco ninguno de ellos cuenta con un actor tan popular como Mario Casas.

Las cuatro películas fueron activas en Twitter durante sus campañas de comunicación. En el día de su estreno, todas habían realizado entre 300 y 936 tuits, salvo Tengo ganas de ti, que sólo había escrito 66 mensajes.

Se evidencia que el número total de tuits emitidos no equivale a un aumento de seguidores. Como se observa con claridad@TGDT escribe muchos menos mensajes, pero logra un impacto mayor que las otras cuentas.

\begin{tabular}{|l|c|c|}
\hline \multicolumn{1}{|c|}{ Usuario } & Personal/Institucional & Impresiones \\
\hline @Cinemex & $\mathrm{I}$ & 577.069 \\
\hline @otramovida & $\mathrm{I}$ & 511.520 \\
\hline @antena3com & $\mathrm{I}$ & 263.964 \\
\hline @3MSC & $\mathrm{I}$ & 205.730 \\
\hline @feas_mc & $\mathrm{P}$ & 177.516 \\
\hline @mariavalverder & $\mathrm{P}$ & 171.393 \\
\hline @Lasextatv & $\mathrm{I}$ & 138.722 \\
\hline @Arturorequejo & $\mathrm{P}$ & 136.404 \\
\hline @Mariocasasweb & $\mathrm{P}$ & 100.934 \\
\hline @danna_mishell & $\mathrm{P}$ & 70.881 \\
\hline
\end{tabular}

Tabla 5. Líderes según impresiones

Para entender el verdadero alcance de las obras y estudiar una posible predicción de resultados de taquilla por medio de Twitter, se utilizó la recaudación total de los cuatro largometrajes, ofrecida por BoxOfficeMojo. Se indica la posición según recaudación entre las películas (nacionales y extranjeras) estrenadas en España durante 2012.

Como se observa la película central de este estudio tiene unos excelentes resultados de público. Se sitúa en el séptimo puesto de la taquilla española a 31 de diciembre de 2012. Los otros tres largometrajes muestran una recaudación muy inferior. Curiosamente, Impávido, que tenía el doble de seguidores que Lobos de Arga obtiene tan sólo algo más de la mitad de la recaudación que ésta. Es decir, el caso de las películas de control muestra cómo el comportamiento en Twitter y el número de seguidores no corresponden directamente a una determinada cantidad de espectadores y por ello se descarta, a día de hoy, la utilización de esta red social como medio de predicción de resultados de taquilla.

Sin duda, hay que valorar que el éxito de Tengo ganas de ti se debe a más factores que a su campaña de comunicación en Twitter. Este largometraje contó con una campaña tanto tradicional como en redes sociales, y a diferencia de los otros tres títulos era una secuela de una película con éxito, y se benefició de un mayor nicho de mercado.

\begin{tabular}{|c|c|c|c|c|c|c|c|c|c|c|c|}
\hline \multirow{2}{*}{$\begin{array}{l}\text { Días para el } \\
\text { estreno }\end{array}$} & \multicolumn{2}{|c|}{ @losninosalvajes } & \multicolumn{2}{|c|}{ @elsnensalvatges } & \multirow{2}{*}{$\begin{array}{c}\Sigma \\
\text { Seg. }\end{array}$} & \multicolumn{2}{|c|}{ @LobosdeArga } & \multicolumn{2}{|c|}{ @Impavido_lapeli } & \multicolumn{2}{|c|}{$@ T G D T$} \\
\hline & Tuits & Seg. & Tuits & Seg. & & Tuits & Seg. & Tuits & Seg. & Tuits & Seg. \\
\hline-5 & 236 & 885 & 414 & 1.986 & 2.871 & 234 & 305 & 205 & 343 & 62 & 49.682 \\
\hline-4 & 245 & 894 & 481 & 2.007 & 2.901 & 254 & 330 & 215 & 432 & 62 & 50.076 \\
\hline-3 & 252 & 904 & 507 & 2.044 & 2.948 & 265 & 336 & 237 & 482 & 63 & 50.527 \\
\hline-2 & 261 & 912 & 518 & 2.068 & 2.980 & 280 & 345 & 265 & 501 & 63 & 51.076 \\
\hline-1 & 273 & 925 & 555 & 2.088 & 3.013 & 299 & 352 & 278 & 523 & 64 & 51.755 \\
\hline 0 & 294 & 936 & 616 & 2.120 & 3.056 & 314 & 366 & 293 & 534 & 66 & 52.413 \\
\hline 1 & 297 & 947 & 625 & 2.152 & 3.099 & 322 & 379 & 296 & 545 & 66 & 54.774 \\
\hline 2 & 309 & 954 & 647 & 2.190 & 3.144 & 337 & 383 & 302 & 559 & 66 & 55.981 \\
\hline 3 & 311 & 954 & 654 & 2.195 & 3.149 & 344 & 386 & 313 & 579 & 66 & 56.835 \\
\hline 4 & 317 & 964 & 663 & 2.218 & 3.182 & 347 & 390 & 322 & 575 & 66 & 57.523 \\
\hline 5 & 321 & 972 & 668 & 2.236 & 3.208 & 348 & 391 & 326 & 578 & 67 & 58.466 \\
\hline 6 & 333 & 976 & 681 & 2.249 & 3.225 & 349 & 392 & 330 & 583 & 67 & 58.900 \\
\hline 7 & 334 & 983 & 694 & 2.266 & 3.249 & 352 & 390 & 332 & 584 & 67 & 59.341 \\
\hline 8 & 335 & 986 & 695 & 2.278 & 3.264 & 353 & 394 & 332 & 584 & 67 & 59.981 \\
\hline 9 & 337 & 990 & 696 & 2.290 & 3.280 & 354 & 396 & 332 & 584 & 67 & 60.346 \\
\hline 10 & 340 & 998 & 700 & 2.299 & 3.297 & 354 & 397 & 332 & 584 & 68 & 60.611 \\
\hline
\end{tabular}

Tabla 6. Control de seguidores 5 días antes y 10 días después del estreno de cada película 


\section{Conclusiones}

Esta investigación ha permitido observar y cuantificar el flujo de tuits, número de seguidores y evolución del factor Klout de los perfiles implicados en la campaña comunicativa de Tengo ganas de ti.

La presencia en torno a dicha película es significativamente alta en Twitter. Los perfiles de los actores protagonistas y los oficiales consiguen un importante aumento de seguidores. Se pueden considerar líderes de opinión. Se observa que los tuits realizados por estos perfiles son retuiteados con frecuencia por otros usuarios. Del mismo modo, las cuentas institucionales participan en la formación de debates y generan expectativas sobre el largometraje. Pero es @mario_ casas_quien muestra una mayor influencia entre el público potencial de la obra. Cada mensaje de este usuario logra un altísimo impacto.

Los perfiles de los protagonistas ganan más seguidores diarios que los de las películas

La campaña comunicativa en Twitter de Tengo ganas de ti creó una red entre todos los perfiles implicados en el lanzamiento, aprovechando así la retroalimentación entre seguidores. Todos los usuarios oficiales y los protagonistas aumentaron sus seguidores y sus factores Klout.

Se ha estudiado la predicción de taquilla por medio de Twitter. Los datos de Tengo ganas de ti y los de las películas de control no permiten elaborar un sistema de predicción de resultados de público mediante Twitter. Aun sin poder presentar este sistema de predicción de taquilla, el estudio comparado con las otras tres obras revela que la ausencia en Twitter de un debate o una actividad significativa, augura un escaso rédito comercial para la película. Además, el éxito de la estrategia promocional de Tengo ganas de ti parece avalar que existe una relación entre el aumento de actividad en Twitter y el mayor número de espectadores cinematográficos.

Por último, como demuestra el caso de Tengo ganas de ti, la actividad en Twitter es potencialmente tan expansiva que justifica que productoras y distribuidoras apuesten por este sitio para promocionar sus productos pero, siempre, como complemento de una campaña de comunicación más amplia.

\section{Bibliografía}

Fernández, Imma (2012). “Fernando González Molina: Mario tiene esa cosa animal de Marlon Brando". El periódico 22/06/2012. http://www.elperiodico.com/es/noticias/ocio-y-cultura/ fernando-gonzalez-molina-mario-tiene-esa-cosa-animalmarlon-brando-1956430

Ferreras-Rodríguez, Eva-María (2011). "La estrategia de la corporación EITB (Euskal Irratia Telebista) en Facebook y Twitter". Razón y palabra, n. 76, pp. 1-25.

http://www.razonypalabra.org. $m x / N / N 76 / v a r i a / 4 a \% 20$ entrega/36_Ferreras_V76.pdf

García-García, Francisco; Gértrudix, Manuel (2009). “El Mare Nostrum Digital: mito, ideología y realidad de un imaginario sociotécnico". Revista Icono14, n. 12, pp. 7-30. http://www.icono14.net/Num.-12.-Nativos-digitales/elmare-nostrum-digital

Hennig-Thurau, Thorsten; Wiertz, Caroline; Feldhaus, Fabian (2012). "Exploring the "Twitter effect": an investigation of the impact of microblogging word of mouth on consumers' early adoption of new products". Social science research network.

http://ssrn.com/abstract=2016548

Ishii, Akira; Arakaki, Hisashi; Matsuda, Naoya; Umemura, Sanae; Urushidani, Tamiko; Yamagata, Naoya; Yoshida, Narihiko (2012). "The 'hit' phenomenon: a mathematical model of human dynamics interactions as a stochastic process". New journal of physics, n. 14.

http://dx.doi.org/10.1088/1367-2630/14/6/063018

Marwick, Alice; Boyd, Danah (2011). "To see and be seen: celebrity practice on Twitter". Convergence, v. 17, n. 2, pp. 139-158.

http://dx.doi.org/10.1177/1354856510394539

Núñez-Gómez, Patricia; García-Guardia, María-Luisa; Hermida-Ayala, Lourdes-Ainhoa (2012). "Tendencias de las relaciones sociales e interpersonales de los nativos digitales". Revista latina de comunicación social, n. 67, pp. 179-202. http://www.revistalatinacs.org/067/art/952_UCM/08_ Patricia.html

http://dx.doi.org/10.4185/RLCS-067-952-179-206

Said-Hung, Elías; Arcila-Calderón, Carlos (2011). "Los líderes de opinión en Colombia, Venezuela e Irán. El caso de los 20 usuarios más vistos en Twitter". Comunicación y sociedad, v. 24, n. 1, pp. 75-100.

http://www.unav.es/fcom/comunicacionysociedad/ descarga_doc.php?art_id=379

Serrano-Puche, Javier (2012). "Herramientas web para la medición de la influencia digital: análisis de Klout y Peerlndex". El profesional de la información, 2012, mayo-junio, v. 21, n. 3, pp. 298-303. http://dx.doi.org/10.3145/epi.2012.may.11

Zhang, Wenbin; Skiena, Steven-Sol (2009). "Improving movie gross prediction through news analysis". IEEE/WIC/ACM, v. 1, pp. 301-304.

http://dx.doi.org/10.1109/WI-IAT.2009.53 